
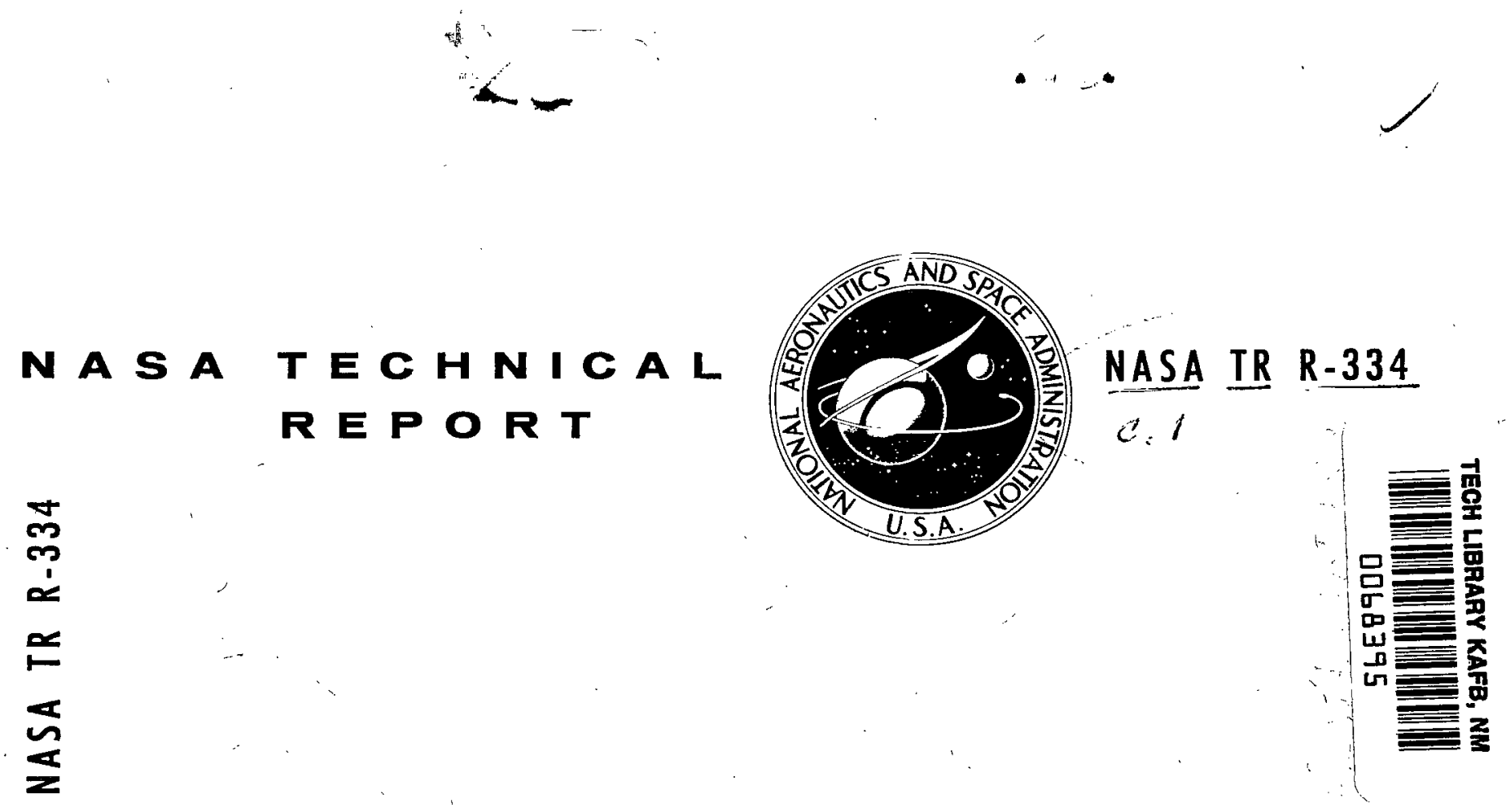

IOAN COPY: RETURA TO AFWT (WLOL) RIRTINAD AFB, N URB

\title{
PHOTON COUNTING: A PROBLEM IN CLASSICAL NOISE THEORY
}

by Sherman Karp and John R. Clark

Electronics Research Center -

Cambridge, Mass.

National aERONAUTICS AND SPACE ADMINISTRATION - WASHINGTON, D. C. - APRIL 1970 
1. Report No.

NASA TR R -334

4. Title and Subtitle

Photon Counting: A Problem In

Classical Noise Theory

7. Author(s)

Sherman Karp and John R. Clark

9. Performing Orgonization Namo and Address

Electronics Research Center

Cambridge, Mass. 02139

12. Sponsoring Agency Name and Address

National Aeronautics and Space Administration

Washington, D.C. 20546

15. Supplementary Notes

16. Absiract this report we formulate the general problem of determining the photoelectron "counting" distribution resulting from an electromagnetic field impinging on a quantum detector. Although the detector model used was derived quantum mechanically, our treatment is wholly classical and includes all results known to-date. This combination is commonly referred to as the semiclassical approach. The emphasis, however, 1ies in directing the problem towards optical communication.

The electromagnetic field is assumed to be the sum of a deterministic signal and a zero-mean, narrow-band, gaussian random process, and is expanded in a Karhunen-Loéve series of orthogonal functions. Several examples are given. It is shown that all the results obtainable can be written explicitly in terms of the noise covariance function. Particular attention is given to the case of a signal plus white gaussian noise, both of which are band-limited to $\pm \mathrm{B} \mathrm{Hz}$. Since the result is a fundamental one, to add some physical insight, we show four methods by which it can be obtained. Various limiting forms of this distribution are derived, including the necessary conditions for those commonly accepted. The likelihood functional is established and is shown to be the product of Laguerre polynomials. For the problem of continuous estimation, the Fisher information kernel is derived and an important limiting form is obtained. The MAP (maximum a posteriori) and ML (maximum likelihood) estimation equations are also derived. In the latter case the results are also functions of Laguerre polynomials.

17. Key Words

- Photon Counting

- Quantum Detection

- Optical Communication

- Noise

19. Security Classif. (of this report)

Unclassified
18. Distribution Statement

Unclassified - Unlimited 


\section{PHOTON COUNTING: A PROBLEM IN}

CLASSICAL NOISE THEORY

By Sherman Karp and John R. Clark Electronics Research Center

\section{SUMMARY}

In this report we formulate the general problem of determining the photoelectron "counting" distribution resulting from an electromagnetic field impinging on a quantum detector. Although the detector model used was derived quantum mechanically, our treatment is wholly classical and includes all results known to-date. This combination is commonly referred to as the semiclassical approach. The emphasis, however, lies in directing the problem towards optical communication.

The electromagnetic field is assumed to be the sum of a deterministic signal and a zero-mean, narrow-band, gaussian random process, and is expanded in a Karhunen-Loéve series of orthogoral functions. Several examples are given. It is shown that all the results obtainable can be written explicitly in terms of the noise covariance function. Particular attention is given to the case of a signal plus white gaussian noise, both of which are bandlimited to $\pm \mathrm{B} \mathrm{Hz}$. Since the result is a fundamental one, to add some physical insight, we show four methods by which it can be obtained. Various limiting forms of this distribution are derived, including the necessary conditions for those commonly accepted. The likelihood functional is established and is shown to be the product of Laguerre polynomials. For the problem of continuous estimation, the Fisher information kernel is derived and an important limiting form is obtained. The MAP (maximum a posteriori) and ML (maximum likelihood) estimation equations are also derived. In the latter case the results are also functions of Laguerre polynomials. 


\section{INTRODUCTION}

Since the advent of the laser, a problem of growing importance in optical communications and coherence theory has been the determiniation of the output statistics of a quantum detector excited by a narrow-band gaussian source. In optical communications, knowledge of these statistics is necessary for the application of the techniques of optimum detection and estimation theory. In the physical theory of coherence, these statistics are a means by which the light incident on the detector can be studied. In both cases a useful statistic, which is relatively easy to evaluate, is the probability $p_{N_{t}}(k)$ of detecting $k$ events, or "counts," in the time interval $(0, t]$. In an idealized detector the conditional probability of $\mathrm{k}$ counts in $(0, t]$, given the incident radiation, can be shown to obey a Poisson law, with the time-averaged intensity of the field as rate parameter.

Although this idealized detector model is based on the quantum theory of photodetection, statistics such as $\mathrm{p}_{\mathrm{N}_{t}}(\mathrm{k})$ can, if desired, be correctly calculated by using only classical tools, provided the proper quantum model has been postulated. This is the essence of the so-called "semiclassical" approach, which we shall use in this report (ref. 1). The value of this approach lies not so much in its freedom from quantum-mechanical subtleties, as much as in the ease with which it allows meaningful physical interpretations to be made and comparisons to be drawn. We shall therefore not dwell on the physical considerations leading to the derivation of the Poisson model, since it is generally accepted as an adequate probabilistic description of an ideal quantum detector localized at a point in space (ref. 2). The physics underlying this model and others is discussed at length in the literature on quantum detectors.

The problem of determining the counting distribution $\mathrm{p}_{\mathrm{N}_{\mathrm{t}}}(\mathrm{k})$ has been approached by a number of authors, within the framework of laser intensity fluctuation studies (refs. 3 through 8 ). It 
is intimately connected with the classic noise theory problem of finding the statistics of the time-averaged square of a real process. Random functionals of this type have been treated in considerable generality for gaussian processes; we list only a few of the more important works in references 9 through 15 .

Here, we present a general method for finding $\mathrm{p}_{\mathrm{N}_{\mathrm{t}}}(\mathrm{k})$ when the light incident on the detector is a deterministic signal plus a narrow-band gaussian process. Our results are shown to encompass as special cases several previously known results. The method is a generalization of Mandel's (ref. 5) in which we allow the incident field to have a nonzero mean, and $\mathrm{p}_{\mathrm{N}_{\mathrm{t}}}(\mathrm{k})$ is expressed in terms of the cumulants of the time-averaged intensity. Several specific examples are worked out in detail, which are in agreement with known results when such results exist. Considerable attention is given to the important case in which the radiation is a deterministic signal in band-limited white gaussian noise. For this example, we show how the results can be applied to some problems of interest in optical communications.

DERIVATION OF THE COUNTING DISTRIBUTION

First, we consider the Poisson model for the ideal quantum detector. Given a counting statistic $N_{t}$ at time $t$, conditioned on a function $[a(\tau) ; 0<\tau \leq t]$, such that it obeys a Poisson law,

$$
\begin{aligned}
\operatorname{Pr}\left\{N_{t}\right. & =k \mid[a(\tau) ; 0<\tau \leq t]\} \\
& =\frac{[m(t)]^{k}}{k !} e^{-m(t)} \\
m(t) & =\alpha \int_{0}^{t}|a(\tau)|^{2} d \tau
\end{aligned}
$$

we want to know the probability $\operatorname{Pr}\left\{\mathrm{N}_{t}=\mathrm{k}\right\}$ when $\mathrm{a}(\tau)$ is the complex envelope of a narrow-band gaussian process. Here $\alpha=n / h \nu$, where 
$\eta$ is the quantum efficiency, $h$ is Planck's constant, and $v$ is the frequency. With $m_{t}$ the random value assumed by $m\left(t^{\prime}\right)$ at time $t^{\prime}=t$, it is clear from Eq. (1) that the probability of $k$ counts in $(0, t]$ is given formally by

$$
\mathrm{p}_{\mathrm{N}_{t}}(\mathrm{k})=\frac{1}{\mathrm{k} !} \mathrm{E}\left\{\mathrm{e}^{-\mathrm{m}_{\mathrm{t}}} \mathrm{m}_{t}^{\mathrm{k}}\right\}
$$

where the expectation is taken over the random variable $\mathrm{m}_{t}$. We shall take $a(\tau)$ to be the complex envelope of a real, not necessarily stationary, gaussian process $\alpha(\tau)$,

$$
\alpha(\tau)=\operatorname{Re}\left[a(\tau) e^{j 2 \pi f} f_{0}\right]
$$

which is assumed to be narrow-band about some very high frequency $f_{0}$. We shall also assume that the covariance function of $a(\tau)$ is real. With these assumptions we can show that the real and imaginary parts of $a(\tau)$ are independent and have the same covariance function. In addition we assume that a $(\tau)$ can be written

$$
a(\tau)=s(\tau)+n(\tau)
$$

where $\mathbf{s}(\tau)$ is a deterministic signal and $n(\tau)$ is a zero-mean gaussian random process.

Instead of evaluating Eq. (3) directly for $p_{N_{t}}(k)$, we shall find the characteristic function of $\mathrm{N}_{t}$ :

$$
\begin{aligned}
M_{N_{t}}(j v) & =E\left\{{ }_{N_{t}} \mid m_{t}(j v)\right\} \\
& =E\left\{e^{m_{t}\left(e^{j v}-1\right)}\right\}
\end{aligned}
$$

Then $\mathrm{p}_{\mathrm{N}_{t}}(\mathrm{k})$ can be found either by direct inversion of $\mathrm{M}_{\mathrm{N}_{t}}(j \mathrm{v})$, or from one of the following formulas: 


$$
\begin{aligned}
& \mathrm{p}_{\mathrm{N}_{t}}(\mathrm{k})=\frac{(-1)^{\mathrm{k}}}{\mathrm{k} !} \frac{\partial^{\mathrm{k}}}{\partial \xi^{\mathrm{k}}} \mathrm{M}_{\mathrm{N}_{t}}[\ln (1-\xi)] \xi=1 \\
& \mathrm{p}_{\mathrm{N}_{t}}(\mathrm{k})=\frac{1}{\mathrm{k} !} \sum_{n=0}^{\infty} \frac{(-1)^{\mathrm{n}}}{\mathrm{n} !} \mu_{\mathrm{k}+\mathrm{n}}
\end{aligned}
$$

$\mu_{i}$ is the $i-t h$ moment of $m_{t}$ in terms of $M_{N_{t}}(j v)$,

$$
\mu_{i}=\frac{\partial^{i}}{\partial \xi^{i}} M_{N_{t}}[\ln (1+\xi)] \xi=0
$$

Equation ( 8 ) is the equivalent of differentiating the probability generating function of $\mathrm{N}_{t}$, and $\mathrm{Eq}$. (9) is the result of expanding the exponential in Eq. (3) in a power series.

We see from Eq. (7) that $M_{N_{t}}(j v)$ is simply the moment generating function of $\mathrm{m}{ }^{\prime}$

$$
M_{m_{t}}(u)=E\left\{e^{m_{t}^{u}}\right\}
$$

evaluated at $u=e^{j v}-1$; thus, we can confine our attention to the random variable, $m_{t}$.

It is convenient to expand $a(\tau)$ in a Karhunen-Loéve series on $[0, t]$ (ref. 16):

$$
\begin{aligned}
a(\tau) & =\sum_{i=1}^{\infty} a_{i} \phi_{i}(\tau) \\
& =\sum_{i}\left(s_{i}+n_{i}\right) \phi_{i}(\tau)
\end{aligned}
$$

The equality, of course, is in the sense of "limit-in-the-mean." The coefficients are given by 


$$
\begin{aligned}
& n_{i}=\left(n, \phi_{i}\right) \\
& s_{i}=\left(s, \phi_{i}\right)
\end{aligned}
$$

where

$$
(x, y)=\int_{0}^{t} x(\tau) y^{*}(\tau) d \tau
$$

and the $\left\{\phi_{i}\right\}$ are eigenfunctions of the integral equation

$$
\lambda_{i} \phi_{i}(u)=k_{n} \phi_{i}=\int_{0}^{t} k_{n}(u, v) \phi_{i}(v) d v
$$

Here $\mathrm{K}_{\mathrm{n}}(\mathrm{u}, \mathrm{v})=\mathrm{E}\left\{\mathrm{n}(\mathrm{u}) \mathrm{n}^{*}(\mathrm{v})\right\}$ is the covariance kernel of the noise. The $\left\{\phi_{i}\right\}$ are normalized so that $\left(\phi_{i}, \phi_{j}\right)=\delta_{i j}$.

It is clear from the orthonormality of the eigenfunctions that $\mathrm{m}_{t}$ can be written

$$
m_{t}=\alpha \sum_{i}\left|a_{i}\right|^{2}=\alpha \sum_{i}\left|s_{i}+n_{i}\right|^{2}
$$

Notice that $m_{t}$ is the energy in the process at time $t$. Since $n(\tau)$ is a zero-mean gaussian random process, the $\left\{n_{i}\right\}$ are gaussian random variables, with $E\left(n_{i}\right)=0$ and $E\left(n_{i} n_{j}^{*}\right)=\lambda_{i} \delta_{i j}$. This orthogonality depends critically upon choosing the basis to satisfy Eq. (16) uniquely. If, however, there is no noise $(\mathrm{n}(\tau)=0), \mathrm{a}(\mathrm{t})=\mathrm{s}(\tau)$ can be expanded in any complete orthonormal set $\left\{\psi_{i}\right\}$ on $[0, t]$, and Eq. (16) is irrelevant. For this case, with $c_{i}=\left(a, \psi_{i}\right), E q$. (1) can be written

$$
\begin{aligned}
\operatorname{Pr}\left\{\mathrm{N}_{t}\right. & =k \mid[a(\tau) ; 0<\tau \leq t]\} \\
& =\frac{\left[\sum_{i} \alpha\left|c_{i}\right|^{2}\right]^{k}}{k !} \exp -\sum_{i} \alpha\left|c_{i}\right|^{2}
\end{aligned}
$$


Each coordinate axis in the space contributes an independent Poisson variate $\mathrm{N}_{t i}$ '

$$
\begin{aligned}
\operatorname{Pr}\left\{\mathrm{N}_{t i}\right. & \left.=\mathrm{k}_{i} \mid[\mathrm{a}(\tau) ; 0<\tau \leq t]\right\} \\
& =\operatorname{Pr}\left\{\mathrm{N}_{t i}=\mathrm{k}_{i} \mid \mathrm{c}_{i}\right\}=\frac{\left[\alpha\left|\mathrm{c}_{i}\right|^{2}\right]^{k_{i}}}{k_{i} !} e^{-\alpha\left|c_{i}\right|^{2}}
\end{aligned}
$$

where $N_{t}=\sum_{i} N_{t i}$ and $k=\sum_{i} k_{i}$ (ref. 17). This is clearly independent of the particular basis chosen; only the $\left\{c_{i}\right\}$ change. Each axis always contributes an independent Poisson variate. In addition, since $\sum_{i}\left|c_{i}\right|^{2}$ is the energy of a $(\tau)$ in $[0, t]$, the conditional density of $\mathrm{N}_{t}$ is independent of the functional form of $a(\tau)$. If we choose $\left\{\psi_{i}\right\}$ to be the sinusoidal set on $[0, t]$, then $\left|c_{i}\right|^{2}$ represents the energy of a $(\tau)$ at the frequency of $\psi_{i}$. If $a(\tau)=\psi_{j}(\tau)$ for some $j$, then $k=k_{j}$ and $E q \cdot$ (18) reduces to Eq. (19) with $i=j$.

For the more general case of nonzero noise, we observe that only one particular orthonormal set can be used as a basis for expanding $a(\tau)$, if we desire each axis in the space to contribute an independent variate to $\mathrm{N}_{t}$. That basis, of course, must satisfy $\mathrm{K}_{\mathrm{n}} \phi=\lambda \phi, \mathrm{Eq} \cdot(16)$. (The only exception is white gaussian noise, for which Eq. (16) is satisfied by any complete orthonormal set; then each axis in the space contributes an independent, identically distributed random variable.)

For narrow-band gaussian noise, we now show that the contribution from each axis is an independent Laguerre-distributed variate and, consequently, that $\mathrm{N}_{t}$ can always be represented as the sum of independent Laguerre random variables.

The real and imaginary parts of $n_{i}=\left(n, \phi_{i}\right)$ are independent, each with variance $\lambda_{i} / 2$, thus (ref. 18, p. 196), 


$$
p_{\left|a_{i}\right|}(A)=\left\{\begin{array}{l}
\frac{2 A}{\lambda_{i}} e^{-\frac{\left|s_{i}\right|^{2}+A^{2}}{\lambda_{i}}} I_{\circ}\left(2 \frac{\left|s_{i}\right|}{\lambda_{i}} A\right), A \geq 0 \\
0, \text { elsewhere }
\end{array}\right.
$$

the so-called Rician density, where $I_{0}$ is the zero-order modified Bessel function of the first kind. It follows that $\left|a_{i}\right|^{2}$ has the density function

$$
p_{\left|a_{i}\right|^{2}}(A)=\left\{\begin{array}{l}
\frac{1}{\lambda_{i}} e^{-\frac{\left|s_{i}\right|^{2}+A}{\lambda_{i}}} I_{\circ}\left(2 \frac{\left|s_{i}\right|}{\lambda_{i}} A^{1 / 2}\right), A \geq 0 \\
0, \text { elsewhere }
\end{array}\right.
$$

Since the $\left\{a_{i}\right\}$ are independent, it follows that the $\left\{\left|a_{i}\right|^{2}\right\}$ are also independent. Thus, from Eqs. (11) and (17) we see that

$$
M_{m_{t}}(u)=\prod_{i} E\left\{e^{\alpha\left|a_{i}\right|^{2} u}\right\}
$$

Now, with $x$ a real, gaussian variate, $E\left\{e^{x^{2} u}\right\}$ is given by (ref. 19, p. 396)

$$
E\left\{e^{x^{2} u}\right\}=\frac{\exp \left[\frac{E^{2}(x) u}{1-2 \operatorname{var}(x) u}\right]}{[1-2 \operatorname{var}(x) u]^{1 / 2}},\left(\operatorname{Re} u<\frac{1}{2 \operatorname{var}(x)}\right)
$$

Thus,

$$
M_{m_{t}}(u)=\prod_{i} \frac{1}{1-\alpha \lambda_{i} u} \exp \frac{\alpha\left|s_{i}\right|^{2} u}{1-\alpha \lambda_{i} u},\left(\operatorname{Re} u<\frac{1}{\alpha \max _{i} \lambda_{i}}\right)
$$


and

$$
M_{N_{t}}(j v)=\prod_{i}\left\{\frac{1}{1-\alpha \dot{\lambda}_{i}\left(e^{j v}-1\right)} \exp \frac{\alpha\left|s_{i}\right|^{2}\left(e^{j v}-1\right)}{1-\alpha \lambda_{i}\left(e^{j v}-1\right)}\right\}
$$

The quantity within the brackets is the characteristic function of a Laguerre random variable for which the probability distribution is (ref. 20)

$$
\mathrm{p}_{N_{t i}}\left(k_{i}\right)=\frac{\left[\alpha \lambda_{i}\right]^{k_{i}}}{\left(1+\alpha \lambda_{i}\right)^{1+k_{i}}} e^{-\frac{\alpha\left|s_{i}\right|^{2}}{1+\alpha \lambda_{i}}} L_{k_{i}}\left(-\frac{\left|s_{i}\right|^{2}}{\lambda_{i}\left(1+\alpha \lambda_{i}\right)}\right)
$$

$\mathrm{L}_{\mathrm{k}}$ is the zero-order Laguerre polynomial (ref. 21, Eq. (8.97)). Thus, we see that $\mathrm{N}_{t}$ can be represented as the sum $\sum \mathrm{N}_{t i}$ of Laguerre variates, as asserted. If the noise is gaussian, the $e_{\perp}$ always exists an operation which acts on Eq. (18), producing another independent coordiniate system of orthogonal axes (Eqs. (25) and (26)).

$$
\begin{aligned}
& \text { If } s_{i}=0 \text { for some } i, \\
& \mathrm{p}_{N_{t i}}\left(k_{i}\right)=\frac{\left[\alpha \lambda_{i}\right] k_{i}}{\left(1+\alpha \lambda_{i}\right)^{1+k_{i}}}
\end{aligned}
$$

Hence any coordinate $\phi_{i}$ for which $s_{i}=0$ contributes a BoseEinstein random variable to $\mathrm{N}_{\mathrm{t}}$.

Proceeding formally, we note that $p_{N_{t}}(k)$ is just the infinite convolution of the $\left\{\mathrm{p}_{\mathrm{N}_{t i}}\left(\mathrm{k}_{\mathrm{i}}\right)\right\}$ for all $i$. As this is usually difficult to evaluate explicitly by using Eq. (26), we shall concentrate our attention on Eq. (24) instead. 
We mention in passing that if the noise $n(\tau)$ is not gaussian, the interpretation in the preceding three paragraphs cannot be used; i.e., there does not exist an orthonormal basis in which $a(\tau)$ can be expanded such that $N_{t}$ can be represented as the sum of independent variates.

$\mathrm{M}_{\mathrm{m}_{t}}(\mathrm{u})$, Eq. (24), can be cast into a completely equivalent form that does not explicitly involve the eigenvalues $\left\{\lambda_{i}\right\}$ (refs. 9 and 22). The identities which make this possible are (ref. 23)

$$
\sum_{i} \lambda_{i}^{k}=\operatorname{Tr} k_{n}^{(k)}=\int_{0}^{t} k_{n}^{(k)}\left(t^{\prime}, t^{\prime}\right) d t^{\prime}
$$

and

$$
\sum_{i}\left|s_{i}\right|^{2} \lambda_{i}^{k}=\left(s, k_{n}^{(k)} s\right)
$$

where

$$
\begin{aligned}
& K_{n}^{(k)}(u, v)=\int_{0}^{t} K_{n}^{(k-1)}(u, \xi) K_{n}(\xi, v) d \xi \\
& K_{n}^{(1)}=K_{n} \\
& K_{n}^{(0)} \equiv \text { identity operator }
\end{aligned}
$$

By expanding $\log \left(1-\alpha \lambda_{i} u\right)$ and $\left(1-\alpha \lambda_{i} u\right)^{-1}$ in power series, and using Eqs. (28) and (29), we get the immediate result

$$
M_{m_{t}}(u)=\exp \sum_{k=1}^{\infty} \frac{k_{k}}{k !} u^{k}, \quad\left(\operatorname{Re} u<\frac{1}{\alpha \max _{i}\left(\lambda_{i}\right)}\right)
$$

and $\left\{k_{i}\right\}$, the cumulants of $\mathrm{m}_{t}$, are

$$
k_{i}=\left[(i-1) ! \operatorname{Tr} k_{n}^{(i)}+i !\left(s, k_{n}^{(i-1)} s\right)\right] \alpha^{i}
$$


For the case, $s(\tau) \equiv 0$, a number of authors have obtained expressions equivalent to Eq. (24) or Eq. (33) (refs. 3, 4, 7, and 9). Related results have also been obtained for real, rather than complex, $a(\tau)$ (refs. 9, 10, 11, 12, 14, and 15).

The form of the noise will often dictate which of the two formulas, Eq. (24) or Eq. (33), will be the more convenient in practice. The integral equation (Eq. (16)) is difficult to solve, and even when the eigenvalues are available, Eq. (24) might not simplify significantly. On the other hand, as Slepian (ref. 1l) has pointed out, the iterated kernels (Eq. (30)) are usually difficult to evaluate beyond the first few orders; however, they are often all that is needed for a good approximation.

We can now find $\mathrm{p}_{\mathrm{N}_{\mathrm{t}}}(\mathrm{k})$ by using one of the formulas, Eq. (8) or Eq. (9). It turns out that Eq. (9) can be evaluated almost trivially with the help of the moment generating function $\mathrm{M}_{\mathrm{m}_{t}}(u)$. Since the moments $\left\{\mu_{i}\right\}$ and the cumulants $\left\{\kappa_{i}\right\}$ are related, we need not evaluate Eq. (10) directly. We use instead the formula (ref. 24),

$$
\mu_{k}=\sum_{i=1}^{k} \frac{1}{i !} \sum_{\substack{i=1 \\ \sum_{j=1} k_{j}=k}} \frac{k !}{k_{1} ! \cdots k_{i} !} k_{k_{1}} k_{k_{2}} \cdots k_{k_{i}}
$$

Along with Eqs. (34) and (35), Eq. (9) gives an exact expression for $\mathrm{p}_{\mathrm{N}_{\mathrm{t}}}(\mathrm{k})$, the desired counting distribution, in terms of the covariance $\mathrm{K}_{\mathrm{n}}$ and the signal s.

The fundamental quantities here are the cumulants $\left\{\kappa_{i}\right\}$, in terms of which a number of the statistical characteristics of $\mathrm{N}_{t}$ can be expressed. Some of the more important relations are derived in the Appendix. Using Eqs. (28), (29), (34), and (A2), we find the mean and variance of $\mathrm{N}_{t}$ to be 


$$
\begin{aligned}
E\left(N_{t}\right)= & \sum_{i} \alpha \lambda_{i}+\alpha(s, s) \\
= & \alpha \operatorname{Tr} k_{n}+\alpha(s, s) \\
\operatorname{var}\left(N_{t}\right)= & \sum_{i} \alpha \lambda_{i}+\alpha(s, s)+\sum_{i}\left[\alpha \lambda_{i}\right]^{2} \\
& +2 \sum_{i}\left[\alpha \lambda_{i}\right] \alpha\left|s_{i}\right|^{2} \\
= & \alpha \operatorname{Tr} k_{n}+\alpha(s, s)+\alpha^{2} \operatorname{Trk}_{n} \\
& +2 \alpha^{2}\left(s, k_{n} s\right)
\end{aligned}
$$

Notice that the only quantities involved in Eqs. (36) and (37) are the noise covariance, the signal, and the time, $t$.

It should be emphasized that our method of finding $p_{N_{t}}(k)$ through the characteristic function is not always the easiest path to the desired answer. Indeed, for certain noise sources the most direct route to $p_{N_{t}}(k)$ might be the direct evaluation of $\mathrm{p}_{\mathrm{m}_{t}}(\mathrm{M})$, the density function of $\mathrm{m}_{t^{\prime}}$ and use of Eq. (3). Whichever method is to be used is best decided when the precise form of $\mathrm{K}_{\mathrm{n}}$ has been ascertained.

\section{EXAMPLES AND APPLICATIONS}

It can be seen that the mathematical form of $p_{N_{t}}(k)$ is considerably more complicated than that of $\mathrm{M}_{\mathrm{N}_{t}}(j v)$ - not a surprising situation in nonlinear noise problems. In most applications of interest, one expects that $\mathrm{p}_{\mathrm{N}_{t}}(\mathrm{k})$ will be much easier to find by evaluating $\mathrm{M}_{\mathrm{N}_{t}}(\mathrm{jv})$ first, rather than by going directly to Eqs. (9), (34), and (35). In the examples to follow, this is certainly the case. 


$$
\text { Noise only }(s(\tau) \equiv 0)
$$

Integrated white noise: $k_{n}(u, v)=\rho^{2} \min (u, v)$.- Although this example appears to be only of academic value, it is illustrative of a particularly simple means of obtaining $p_{N_{t}}(k)$. It is well known that the eigenvalues of $\mathrm{Eq}$. (16) are (ref. 19, page 196)

$$
\lambda_{i}=\frac{4 \rho^{2} t^{2}}{\pi^{2}(2 i-1)^{2}}, i=1,2,3, \ldots
$$

Inserting this in Eq. (24) and using a tabulated product rule, we get (ref. $21, \mathrm{Eq} .(1.4313)$ )

$$
M_{t}(u)=\sec (\rho t \sqrt{\alpha u})
$$

The coefficients in the Taylor series expansion of Eq. (39) are the moments $\left\{\mu_{i}\right\}$ of $m_{t}$; since (ref. 2l, Eq. (1.4119))

$$
\sec x=\sum_{k=0}^{\infty} \frac{\left|E_{2 k}\right|}{(2 k) !} x^{2 k},\left(x^{2}<\frac{\pi^{2}}{4}\right)
$$

we have

$$
\mu_{k}=\frac{k !}{(2 k) !}\left|E_{2 k}\right|\left(\alpha \rho^{2} t^{2}\right)^{k}
$$

and the $\left\{E_{i}\right\}$ are the Euler numbers. Equation (9) now gives $p_{N_{t}}(k)$ directly:

$$
p_{N_{t}}(k)=\sum_{n=0}^{\infty} \frac{(-1)^{n}}{(2 k+2 n) !}\left(\begin{array}{c}
k+n \\
n
\end{array}\right)\left|E_{2 k+2 n}\right|\left(\alpha \rho^{2} t^{2}\right)^{k+n}
$$

Because of the simple form of $\mathrm{M}_{\mathrm{m}}(u)$, we can find the counting distribution without first having to compute the cumulants $\left\{\kappa_{i}\right\}$. The mean and variance of the counts work out to be 


$$
E\left(N_{t}\right)=\frac{\alpha}{2} \rho^{2} t^{2}
$$

$$
\operatorname{var}\left(N_{t}\right)=\frac{\alpha}{2} \rho^{2} t^{2}+\frac{\alpha^{2}}{6} \rho^{4} t^{4}
$$

First-order Markov noise: $\mathrm{K}_{\mathrm{n}}(\mathrm{u}, \mathrm{v})=\mathrm{P} \exp -\beta|\mathrm{u}-\mathrm{v}| \cdot-$ Equation (24) becomes

$$
M_{m_{t}}(u)=\prod_{i} \frac{1}{1-\alpha \lambda_{i} u}
$$

with $\lambda_{i}$ a solution of a transcendental equation (ref. 9). Equation (45) has been evaluated by an indirect technique (ref. 14) and is given by

$$
\begin{aligned}
M_{m_{t}}(u) & =e^{\beta t}\left\{\cosh \left[\beta t\left(1-\frac{2 P \alpha}{\beta} u\right)\right]\right. \\
& \left.+\frac{1-\frac{P \alpha}{\beta} u}{1-\frac{2 P \alpha}{\beta} u} \sinh \left[\beta t\left(1-\frac{2 P \alpha}{\beta} u\right)\right]\right\}^{-1}
\end{aligned}
$$

This does not easily yield a useful expression for $\mathrm{p}_{\mathrm{t}}(\mathrm{k})$; however, using the Leibnitz differentiation rule, Bedard (ref. 3) has obtained recurrence relations for the counting distribution and its factorial moments. For certain limiting cases, approximate counting distributions have been obtained (ref. 7).

The mean and variance of $\mathrm{N}_{t}$ are easily found to be

$$
E\left(N_{t}\right)=\alpha P t
$$

$$
\operatorname{var}\left(N_{t}\right)=\alpha P t+\frac{(\alpha P)^{2}}{2 \beta^{2}}\left[2 \beta t+e^{-2 \beta t}-1\right]
$$

Band-limited white gaussian noise.- Let $\mathrm{n}(\tau)$ be a white gaussian process, band-limited to $\pm \mathrm{B} \mathrm{Hz}$, with two-sided spectral 
height $\mathrm{N}_{\mathrm{O}^{-}}$Taking the first $2 \mathrm{Bt}+1$ eigenvalues of Eq. (16) to be the same $\left(\mathrm{N}_{0}\right)$, and the rest to be zero (ref. 19, page 193), Eq. (24) becomes

$$
M_{m_{t}}(u)=\left(\frac{1}{1-\alpha N_{0} u}\right)^{2 B t+1}
$$

that is,

$$
M_{N_{t}}(j v)=\left[\frac{1}{1-\alpha N_{0}\left(e^{j v}-1\right)}\right]^{2 B t+1}
$$

This can be recognized as the characteristic function of the negative binomial distribution (ref. 17)

$$
\mathrm{p}_{\mathrm{N}_{t}}(\mathrm{k})=\left(\begin{array}{c}
2 \mathrm{Bt}+\mathrm{k} \\
\mathrm{k}
\end{array}\right)\left(\frac{1}{1+\alpha \mathrm{N}_{\mathrm{o}}}\right)^{2 \mathrm{Bt}+1}\left(\frac{\alpha \mathrm{N}_{\mathrm{o}}}{1+\alpha \mathrm{N}_{\mathrm{o}}}\right)^{\mathrm{k}}
$$

If $2 \mathrm{Bt} \ll 1\left(t<\frac{1}{2 \mathrm{~B}}\right)$, only one eigenvalue of Eq. (16) is significant, and Eq. (50) reduces to

$$
M_{N_{t}}(j v)=\frac{1}{1-\alpha N_{0}\left(e^{j v}-1\right)}
$$

In other words, the counting distribution is Bose-Einstein:

$$
\mathrm{p}_{\mathrm{N}_{\mathrm{t}}}(\mathrm{k})=\frac{1}{1+\alpha \mathrm{N}_{\mathrm{o}}}\left(\frac{\alpha \mathrm{N}_{\mathrm{o}}}{1+\alpha \mathrm{N}_{\mathrm{o}}}\right)^{\mathrm{k}}
$$

in agreement with Eq. (27). For $2 \mathrm{Bt} \gg 1$, and $\alpha \mathrm{N}_{0} \ll 1$, it can easily be shown that $\mathrm{p}_{\mathrm{N}_{\mathrm{t}}}(\mathrm{k})$ approaches a Poisson distribution. * on the other hand, for a nonzero signal and no noise, $\mathrm{p}_{\mathrm{N}_{t}}(\mathrm{k})$ is also Poisson, as we have seen. This behavior, in the case of band-limited white-gaussian noise, is due to the complete

* Note: This limiting form can also be shown to be true for firstorder Markov noise, Eq. (46), when $\beta t \gg 1$ and $\beta \gg 4 \mathrm{P} \alpha$. 
"smoothing out" of the intensity fluctuations, while in the signal only case, it is due to the deterministic nature of the signal.

\section{Signal Plus Noise}

Now we remove the restriction that $s(\tau)$ be zero. Assume that $\mathrm{n}(\tau)$ is band-limited white gaussian noise, as above. In addition, assume that $s(\tau)$ is band-limited to $\pm B^{\prime} \mathrm{Hz}, B^{\prime} \leq B$. Equation (25) applies with $\lambda_{i}=N_{0}$, and $p_{N_{t}}(k)$ is just the $(2 \mathrm{Bt}+1)$-fold convolution of the Laguerre density (Eq. (26)) with itself. Omitting the details, we note that the result is (ref. 21, Eq. (8.9771))

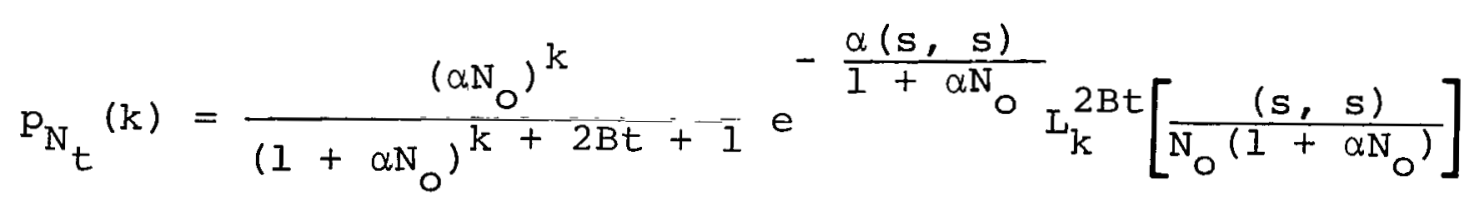

where $\mathrm{L}_{\mathrm{k}}^{2 \mathrm{Bt}}$ is the 2Bt-order Laguerre polynomial. The mean and variance of $\mathrm{N}_{t}$ are found to be

$$
\begin{aligned}
& E\left(N_{t}\right)=(2 B t+1) \alpha N_{0}+\alpha(s, s) \\
& \operatorname{var}\left(N_{t}\right)=(2 B t+1)\left(1+\alpha N_{0}\right) \alpha N_{0}+\left(1+2 \alpha N_{0}\right) \alpha(s, s)
\end{aligned}
$$

Equation (54) could have been found by evaluating $\mathrm{p}_{\mathrm{m}_{t}}$ (M) first, and using Eq. (3) - the alternative approach suggested earlier. For this example, $\mathrm{P}_{\mathrm{m}}$ (M) turns out to be a "noncentral chi-square" density (i.e., the density of the sum of $2 \mathrm{Bt}+1$ independent Ricean variates):

$$
\mathrm{p}_{\mathrm{m}_{t}}(M)=\left\{\begin{array}{l}
\frac{I}{\mathrm{~N}_{0} \alpha}\left[\frac{M}{(s, s) \alpha}\right]^{B t} e^{-\frac{M+(s, s) \alpha}{\alpha N_{O}}} I_{2 B t}\left(\frac{2(s, s)^{1 / 2}}{\alpha^{I / 2} N_{O}} M^{1 / 2}\right) \\
0, \text { elsewhere } \\
M>0
\end{array}\right.
$$


$\mathrm{PN}_{t}(\mathrm{k}), \mathrm{Eq} \cdot(54)$, results when the expression

$$
\int_{0}^{\infty} \frac{1}{k !} M^{k} e^{-M} p_{m_{t}}(M) d M
$$

is evaluated.

For $2 \mathrm{Bt} \ll 1, \mathrm{Eq} .(54)$ reduces to $\mathrm{Eq} .(26)$; for $2 \mathrm{Bt}>>1$, however, $\mathrm{p}_{\mathrm{N}_{\mathrm{t}}}(\mathrm{k})$ approaches a Poisson distribution when $\alpha \mathrm{N}_{\mathrm{O}}$ is small. This is clear from the following discussion.

Let $\alpha \mathrm{N}_{\mathrm{O}} \ll 1$ and $2 \mathrm{Bt} \gg 1$; then, asymptotically,

$$
\begin{aligned}
& \left(\frac{1}{1+\alpha N_{O}}\right)^{2 B t+1} \sim e^{-2 B t \alpha N_{O}} \\
& \frac{\alpha(s, s)}{1+\alpha N_{O}} \sim \alpha(s, s) \\
& \frac{\alpha N_{O}}{1+\alpha N_{O}} \sim \alpha N_{O} \\
& L_{k}^{2 B t}\left[-\frac{(s, s)}{N_{0}\left(1+\alpha N_{0}\right)}\right] \sim \frac{1}{k !}\left(2 B t+\frac{(s, s)}{N_{O}}\right)^{k}
\end{aligned}
$$

These Equations, together with Eq. (54), yield

$$
\mathrm{p}_{N_{t}}(k) \sim \frac{\left[\alpha(s, s)+2 B t \alpha N_{O}\right]^{k}}{k !} e^{-\left[\alpha(s, s)+2 B t \alpha N_{0}\right]}
$$

Thus for small $\alpha \mathrm{N}_{\mathrm{O}}$ and large 2Bt, the counting distribution is Poisson, with the rate parameter the sum of a signal intensity and an independent noise intensity. Expressing this in another way, we note that $\mathrm{N}_{t}$ is the sum of two independent Poisson variates; one associated with the signal (intensity $\frac{1}{t}(s, s) \alpha$ ), 
and one associated with the noise (intensity $2 \mathrm{BN}_{0} \alpha$ ).

Our results for band-limited gaussian noise are well known in the field of coherence theory (refs. 1, 2, 5, 6, 8, 20, and 25); in optical communications, however, they are just beginning to find application (refs. 26, 27, and 28). In earlier papers the asymptotic form (Eq. (63)) was used, with heuristic justification (refs. 29 and 30 ).

\section{ESTIMATION FOR CONTINUOUS WAVEFORMS}

For band-limited white gaussian noise, simple detection and estimation problems can be solved with relative ease because of the independence of noise samples taken at the Nyquist rate. Assume that the interval $[0, t]$ is broken into $2 \mathrm{Bt}$ equal subintervals, each of length $1 / 2 \mathrm{~B}$ sec. Then the probability of $k_{i}$ detector counts in the i-th subinterval is given by

$$
\frac{\left(\alpha N_{0}\right)^{k_{i}}}{\left(I+\alpha N_{0}\right)^{1+k_{i}}} e^{-\frac{\alpha\left|s_{i}\right|^{2}}{1+\alpha N_{0}}} L_{k_{i}}\left[-\frac{\left|s_{i}\right|^{2}}{N_{0}\left(1+\alpha N_{0}\right)}\right]
$$

where $\left|s_{i}\right|^{2}=\left|s\left(\frac{i}{2 B}\right)\right|^{2} / 2 B$. Moreover, the counts in different subintervals are independent, because the noise samples are independent. Thus the joint probability $\mathrm{p}_{\mathrm{N}}(\underline{\mathrm{k}})$ of the counts in each subinterval is simply the product of probabilities (Eq. (64)):

$$
\underline{p}_{\underline{N}} \underline{(k)}=\prod_{i=1}^{2 B t} \frac{\left(\alpha N_{0}\right)^{k_{i}}}{\left(1+\alpha N_{0}\right)^{1+k_{i}}} e^{-\frac{\alpha\left|s_{i}\right|^{2}}{1+\alpha N_{0}}} L_{k_{i}}\left[-\frac{\left|s_{i}\right|^{2}}{N_{0}\left(1+\alpha N_{0}\right)}\right]
$$

with $\underline{N}=$ vector of $2 \mathrm{Bt}$ counting observables;

$\underline{k}=\left(k_{1}, k_{2}, \ldots, k_{2 B t}\right)$. 
It is important to note that, for this representation to be valid when the signal waveform is band-limited to $\pm \mathrm{B} \mathrm{Hz}$, it is essential that the subintervals be of length precisely $1 / 2 \mathrm{~B}$ sec. Equation (65) suggests that we define the likelihood function $\Lambda(\underline{k} \mid \underline{s})$ to be

$$
\Lambda(\underline{\mathrm{k}} \mid \underline{\mathrm{s}})=\prod_{i=1}^{2 \mathrm{Bt}} \mathrm{e}^{-\frac{\alpha\left|s_{i}\right|^{2}}{1+\alpha \mathrm{N}_{0}}} I_{k_{i}}\left[-\frac{\left|s_{i}\right|^{2}}{\bar{N}_{0}\left(1+\alpha N_{0}\right)}\right]
$$

It is now straightforward to set up decision structures for the multiple-hypothesis detection problem. This has been done elsewhere for a number of signaling schemes (ref. 27), so we shall restrict our attention to the estimation problem.

We start with the assumption that $s$ is a transformation, $\mathbf{s}[\tau, \mathrm{x}(\tau)]$, of some function, $\mathrm{x}(\tau)$, which we wish to estimate; $x$ can be a modulation, a set of parameters, or a single number. As is well known, a necessary condition for $\hat{x}_{\text {MAP }}(\tau)$ to be the maximum a posteriori (MAP) estimator of $x(\tau), 0<\tau \leq t$, is

$$
\nabla_{\mathrm{x}}\left[\ln \Lambda+\operatorname{lnp_{\mathrm {x}}(\tau )}[\mathrm{x}(\tau)]\right]_{\mathrm{x}}=\hat{\mathrm{x}}_{\mathrm{MAP}}=0
$$

where $\nabla_{x}$ is the gradient operator with respect to $x(\tau)$, and $\mathrm{P}_{\mathrm{X}(\tau)}[\mathrm{X}(\tau)]$ is the a priori probability density functional of $x(\tau)$. In the absence of prior information, of course, $\mathrm{Eq} \cdot$ (67) reduces to the maximum likelihood equation,

$$
\left.\nabla_{\mathrm{x}} \ln \Lambda\right|_{\mathrm{x}}=\hat{\mathrm{x}}_{\mathrm{ML}}=0
$$

With some manipulation, $\nabla_{x} \ln \Lambda$ works out to be 


$$
\begin{array}{r}
\nabla_{x} \ln \Lambda=\frac{2 \alpha}{1+\alpha i \bar{N}_{0}} \sum_{i=1}^{2 B t} c\left(k_{i}, s_{i}\right) \operatorname{Re}\left[s_{i}^{*} \frac{\partial s_{i}}{\partial x(\tau)}\right] \\
(0<\tau \leq t)
\end{array}
$$

where

$$
c\left(k_{i}, s_{i}\right)=\frac{1}{\alpha N_{0}} \frac{L_{k_{i}}^{1}-1\left[-\frac{\left|s_{i}\right|^{2}}{N_{0}\left(1+\alpha N_{0}\right)}\right]}{L_{k_{i}}\left[-\frac{\left|s_{i}\right|^{2}}{N_{0}\left(1+\alpha N_{0}\right)}\right]}-1
$$

The problem is now simplified if we consider $x$ as a set of parameters (say, $M$ of them). Then, with $x=\left(x_{1}, x_{2}, \ldots, x_{M}\right)$, Eq. (69) reduces to the set of $M$ expressions:

$$
\begin{array}{r}
\nabla_{x} \ln \Lambda=\frac{2 \alpha}{I+\alpha N_{0}} \sum_{i=1}^{2 B t} c\left(k_{i}, s_{i}\right) \operatorname{Re}\left[s_{i}^{*} \frac{\partial s_{i}}{\partial x_{k}}\right] \\
(k=1,2, \ldots, M)
\end{array}
$$

Further simplication is possible if $s$ is a memoryless mapping; thus, with $x_{i}=x(i / 2 B), E q \cdot$ (69) becomes

$$
\begin{aligned}
\nabla_{x} \ell n \Lambda=\frac{2 \alpha}{1+\alpha N_{0}} c\left(k_{i}, s_{i}\right) \operatorname{Re}\left[s_{i}^{*} \frac{\partial s_{i}}{\partial x_{i}}\right] & \\
& (i=1,2, \ldots, 2 B t)
\end{aligned}
$$

In general, it appears that a search procedure is necessary to find $\hat{x}_{\mathrm{MAP}}$ or $\hat{\mathrm{x}}_{\mathrm{ML}}$.

If we now assume that the a priori density $p_{x}(\dot{\tau})[x(\tau)]$ is gaussian, then Eq. (67) reduces to 


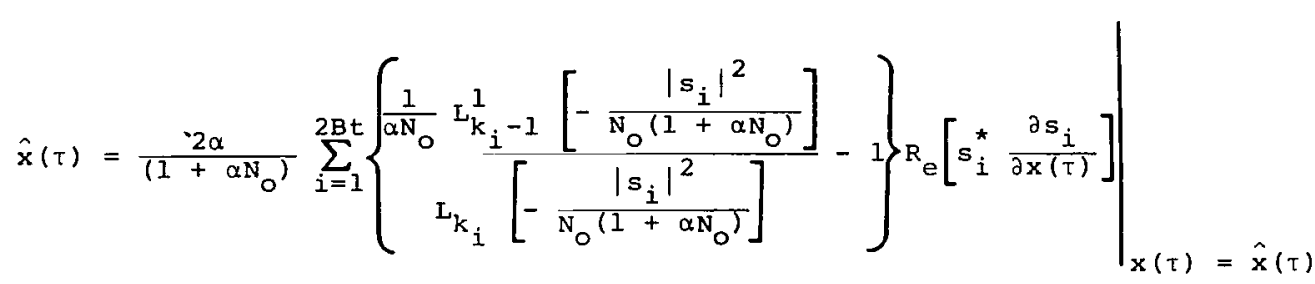

$$
\begin{aligned}
& (0<\tau \leq t)
\end{aligned}
$$

which, as we can see from Figure 1, has very limited use.

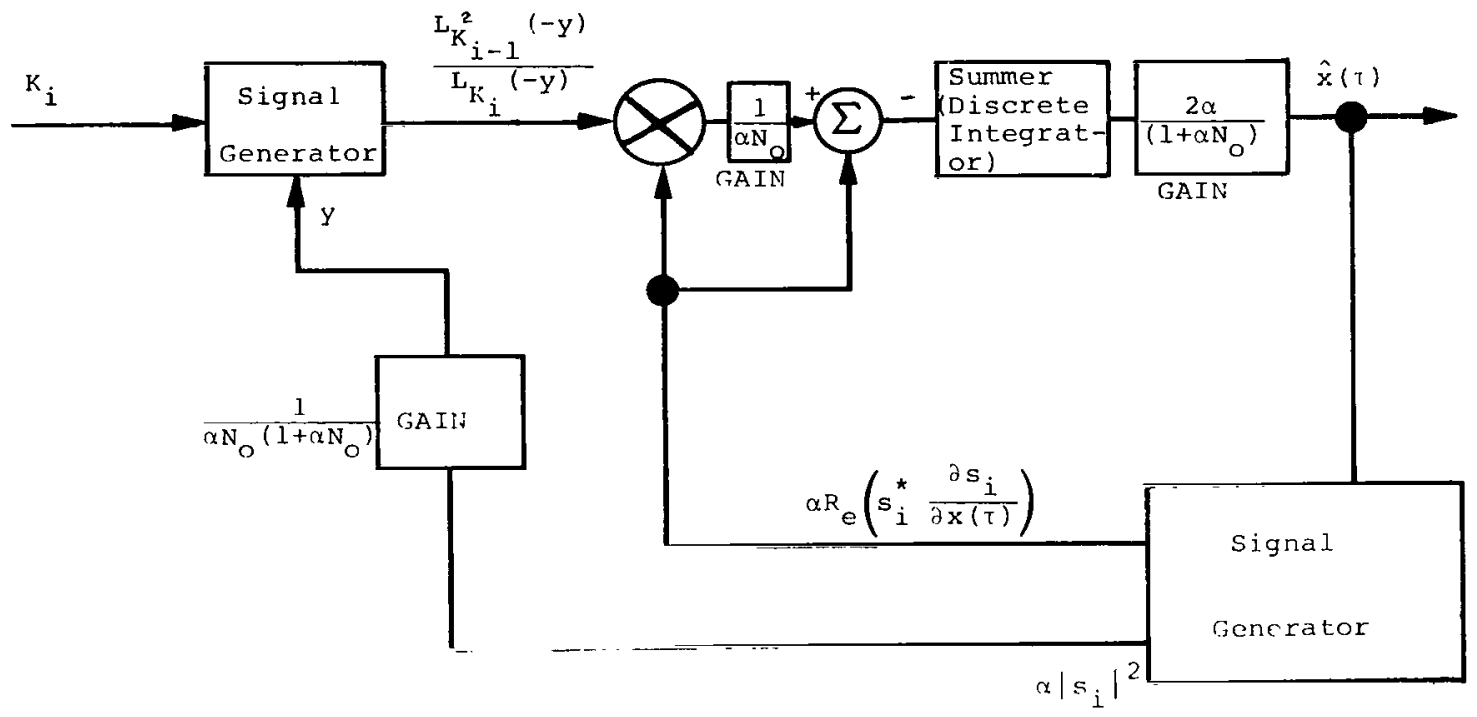

Figure 1.- Schematic of Eq. (73)

However, by making one more assumption, namely that the average noise count per degree of freedom is small:

$$
\alpha N_{0} \ll \min \left[\alpha\left|s_{i}\right|^{2}, 1\right]
$$

Eq. (73) reduces to

$$
\begin{array}{r}
\left.\hat{x}(\tau) \cong \sum_{i=1}^{2 B t}\left[k_{i}-\alpha\left|s_{i}\right|^{2}\right] \frac{\partial}{\partial x(\tau)} \ln \left|s_{i}\right|^{2}\right|_{x=\hat{x}(\tau)} \\
(0<\tau \leq t)
\end{array}
$$

and is shown in Figure 2 . 


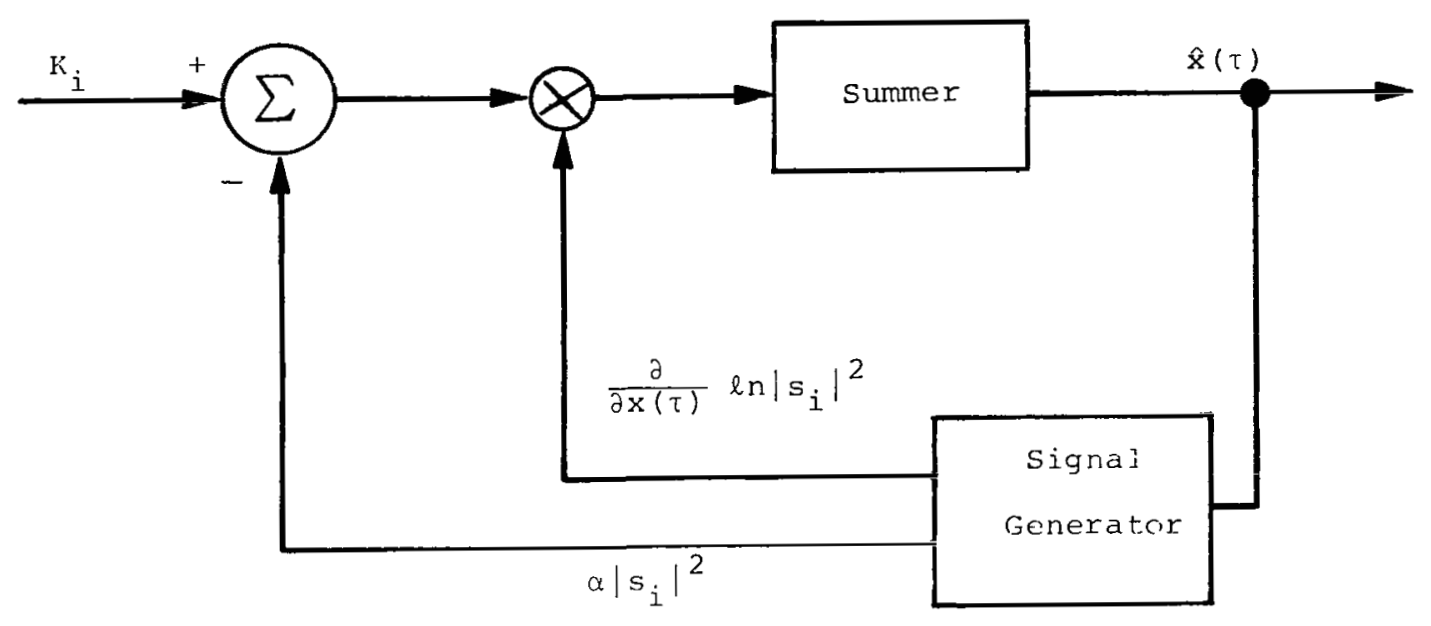

Figure 2.- Schematic of Eq. (75)

Notice that the optimum MAP estimate is performed on the intensity of the process. That is $k_{i}$ is first compared to $\alpha\left|s_{i}\right|^{2}$, the expected count of the received process. Since $\left|s_{i}\right|$ is the envelope of the process, it can be written as $e^{\ell n\left|s_{i}\right|}$, and $\frac{\partial}{\partial \hat{x}} \ln \left|s_{i}\right|$ serves the same function as $\frac{\partial}{\partial \hat{x}} s(t, x)$ in the MAP equations for gaussian systems (ref. 19, p. 432).

If the intensity of the process is constant, such as FM, no phase information can be obtained since $\frac{\partial}{\partial x} \ln \left|s_{i}\right|=0$, making $\hat{\mathrm{x}}=0$. To obtain phase information from an FM or a PM signal, optimal heterodyne detection must be employed. That is, the signal from a local oscillator is aligned and mixed with the received signal over the surface of the detector. Then,

$$
s_{i}=E_{I} e^{j \omega_{1} t+\phi(t)}+E_{L_{0}} e^{j \omega_{2} t}
$$

where $E_{I}$ and $E_{L_{O}}$ are the two electric field strengths, and

$$
\begin{aligned}
\left|s_{i}\right|^{2}=\left|E_{1}\right|^{2} & +\left|E_{L_{O}}\right|^{2}+\left\{E_{1} E_{L_{O}}^{*} e^{\left[j\left(\omega_{1}-\omega_{2}\right) t+\phi\right]}\right. \\
& \left.+E_{1}^{*} E_{L_{O}} e^{-\left[j\left(\omega_{1}-\omega_{2}\right) t+\phi\right]}\right\}
\end{aligned}
$$


Assuming narrow-band signals, we have

$$
\left|s_{i}\right|^{2} \simeq\left|E_{1}\right|^{2}+\left|E_{L_{0}}\right|^{2}+2 E_{1} E_{L_{0}} \cos \left[\left(\omega_{1}-\omega_{2}\right) t+\phi(t)\right]
$$

By making the local oscillator signal large $\left|E_{L_{0}}\right|>>\left|E_{I}\right|$,

$$
\left|s_{i}\right|^{2} \simeq\left|E_{L_{0}}\right|^{2}\left[1+\frac{2 E_{1}}{E_{L_{0}}} \cos \left[\left(\omega_{1}-\omega_{2}\right) t+\phi(t)\right]\right]
$$

and

$$
\ln \left|s_{i}\right|=\frac{1}{2} \ln \left|s_{i}\right|^{2} \simeq \ln \left|E_{L_{0}}\right|+\frac{E_{1}}{E_{L_{0}}} \cos \left[\left(\omega_{1}-\omega_{2}\right) t+\phi(t)\right]
$$

Hence,

$$
\frac{\partial}{\partial x(\tau)} \ln \left|s_{i}\right| \simeq-\frac{E_{1}}{E_{L_{0}}} \frac{\partial \phi}{\partial x(\tau)} \sin \left[\left(\omega_{1}-\omega_{2}\right) t+\phi(t)\right]
$$

We see, then, that the optimum detector is similar, in this case, to a phase-lock loop. It can also be shown by using Eq. (26) and references 18 and 26 that if we consider the random variable $k_{i}$ as a shot-noise process and pass it through a narrow-band filter with bandwidth $2 \mathrm{~W}$ tuned to $\left(\omega_{1}-\omega_{2}\right)$, the density will approach a gaussian density with mean $2 \alpha E_{1} E_{L_{0}} \cos \left[\left(\omega_{1}-\omega_{2}\right) t+\phi(t)\right]$ and variance $\alpha\left|E_{L_{O}}\right| 2 W$.

Using the notion of an information kernel, we can lowerbound the mean-square error of any estimator resulting from Eq. (66), without specifying the form of the estimator (ref. 19, p. 80). Defining

$$
\mathrm{J}_{\mathrm{D}}=\mathrm{E}\left[\frac{\partial \ln \Lambda}{\partial \mathrm{x}(\mathrm{U})} \frac{\partial \ln \Lambda}{\partial \mathrm{x}(\mathrm{v})}\right]
$$




$$
\begin{aligned}
& J_{P}=E\left[\frac{\partial \ln p_{X(\tau)}[X(\tau)]}{\partial x(u)} \frac{\partial \ln p_{X(\tau)}[X(\tau)]}{\partial x(v)}\right] \\
& J_{T}=J_{D}+J_{P}=\text { information kernel }
\end{aligned}
$$

we can show that

$$
\int_{0}^{t}[x(\tau)-\hat{x}(\tau)]^{2} d \tau \geq \operatorname{Tr} J_{T}^{-1}
$$

The expectation in Eqs. (80) and (81) is over the random function, x. Combining Eqs. (69) and (80), we have

$$
\begin{aligned}
J_{D}= & E\left\{\frac{4 \alpha^{2}}{\left(1+\alpha N_{0}\right)^{2}} \sum_{i=1}^{2 B t} \sum_{j=1}^{2 B t} c\left(k_{i}, s_{i}\right)\right. \\
& \left.\cdot c\left(k_{j}, s_{j}\right) \operatorname{Re}\left[s_{i}^{*} \frac{\partial s_{i}}{\partial x(u)}\right] \operatorname{Re}\left[s_{j}^{*} \frac{\partial s_{i}}{\partial x(v)}\right]\right\}
\end{aligned}
$$

To evaluate this further, the transformation $s$ must be specified.

\section{CONCLUSIONS}

We have presented general results for the photoelectron counting distributions arising from the quantum detection of a narrow-band gaussian process. The semiclassical approach has led to probability distributions which could be written explicitly in terms of the covariance function of the gaussian radiation. Four different, but equivalent, methods for evaluating the counting distribution have been presented, the last of which is valid only for white noise. These could be descriptively titled, "the eigenvalue approach," "the iterated kernel approach," "the compound Poisson approach," and "the time-sampling approach." The third method is the one in which Eq. (3) is evaluated directly. Of the four methods, the third and last one lend the 
most physical insight, although the third method is seldom tractable, computationally. The eigenvalue approach will probably find the most use in problems of practical importance.

Several examples have been given, with special emphasis on the important case of a deterministic signal in white gaussian noise. Some limiting forms have been derived, and rigorous justification has been given for the often-made assumption of a Poisson signal in Poisson noise. Finally, the likelihood functional has been defined, and formal answers have been found for the continuous MAP and ML estimation problems, including a simple approximation for low noise, and an expression for the minimum mean-square error of any estimator.

It should be emphasized that, from a communications viewpoint, the detector senses variations in the signal modulus only; all carrier phase information is lost. This does not mean, however, that our results are applicable only to direct (or "energy") detectors. Heterodyne detection can be accomplished simply by illuminating the detector with a local oscillator field; the squared modulus then contains the difference frequency term and the phase information from both the local oscillator and the received field. 
Here we mention a few of the more important statistical quantities associated with the counting distribution, $\mathrm{p}_{\mathrm{N}_{\mathrm{t}}}(\mathrm{k})$. First we note that the factorial moments $\left\{\tilde{u}_{[i]}\right\}^{* *}$ of the counting distribution are equal to the moments $\left\{\mu_{i}\right\}$ of $m_{t}$. This is clear from

$$
\begin{aligned}
\tilde{\mu}_{[n]} & =\sum_{k=n}^{\infty} k(k-1) \ldots(k-n+1) p_{N_{t}}(k) \\
& =\left.(-1)^{n} \frac{\partial^{n}}{\partial \xi^{n}} M_{N_{t}}[\ln (1-\xi)]\right|_{\xi=0}=0 \\
& =\left.(-1)^{n} \frac{\partial^{n}}{\partial \xi^{n}} M_{m_{t}}(-\xi)\right|_{\xi=0} \\
& =\mu_{n} .
\end{aligned}
$$

Also, the cumulants of the counting distribution are connected with the cumulants of $\mathrm{m}_{t}$ by

$$
\tilde{\kappa}_{n}=\sum_{i=1}^{n} A(n, i) \frac{k_{i}}{i !}
$$

where

$$
A(n, i)=\sum_{k=1}^{i}\left(\begin{array}{l}
i \\
k
\end{array}\right)(-1)^{i-k} k^{n}
$$

*The tilde ( $)$ will be used to distinquish moments, etc., of the counting distribution, from corresponding quantities associated with $m_{t}$. 
Equation (A2) is a consequence of differentiating the logarithm of

$$
\begin{aligned}
M_{N_{t}}(j v) & =M_{m_{t}}\left(e^{j v}-1\right) \\
& =\exp \sum_{k=1}^{\infty} \frac{k_{k}}{k !}\left(e^{j v}-1\right)^{k}
\end{aligned}
$$

Similarly, it can be shown that

$$
\tilde{\mu}_{n}=\sum_{i=1}^{n} A(n, i) \frac{\mu_{i}}{i !}
$$

Further, the moments $\left\{\tilde{\mu}_{i}\right\}$ of $N_{t}$ are related to the cumulants $\left\{\tilde{\kappa}_{i}\right\}$ of $N_{t}$ by Eq. (35).

With the help of these formulas and known identities (ref. 24), an exhaustive set of relations can be found for the moments, factorial moments, cumulants, and factorial cumulants of $\mathrm{N}_{t}$ and $\mathrm{m}_{t}$. We shall not, however, reproduce these relations here. 


\section{REFERENCES}

1. Mandel, L., and Wolf, E.: Letter to the Editor. J. Phys. A. (Proc. Phys. Soc.) Ser. 2, vol. 1, 1968, pp. 625-627.

2. Glauber, R. J.: Optical Coherence and Photon statistics. Quantum Optics and Electronics. C. de witt et al., eds. Gordon \& Breach, New York, 1965, pp. 65-185.

3. Bedard, G.: Photon Counting Statistics of Gaussian Light. Phys. Rev., 151, 4, 25 November 1966, pp. 1038-1039.

4. Jakeman, E., and Pike, E. R.: The Intensity-Fluctuation Distribution of Gaussian Light. J. Phys. A. (Proc. Phys. Soc.), 2, 1, 1968, pp. 128-138.

5. Mandel, L.: Fluctuations of Light Beams, Progress in Optics II. E. Wolf, ed. John Wiley \& Sons, Inc., New York, 1963, pp. $181-248$.

6. Glauber, R. J.: Photon Counting and Field Correlations. Physics of Quantum Electronics. P. L. Kelly et al., eds. McGraw-Hill Book Co., Inc., New York, 1965, pp. 788-811.

7. Helstrom, C. W.: The Distribution of Photoelectric Counts from Partially Polarized Gaussian Light. Proc. Phys. Soc., $83,1964, \mathrm{pp} .777-782$.

8. Perina, J.: Determination of the Statistical Properties of Light from Photoelectric Measurements. Czech J. Physics, B17, $1086,1967$.

9. Middleton, D.: Introduction to Statistical Communication Theory. McGraw-Hill Book Co., Inc., New York, 1960.

10. Emerson, R. C.: First Probability Densities for Receivers with Square-Law Detectors. J. Appl. Phys., 24, 1953, p. 1168.

11. Slepian, D.: Fluctuations of Random Noise Power. Bell Sys. Tech. J., 37, 1958, pp. 163-184. 
12. Kac, M., and Siegert, A. J. F.: On the Theory of Noise in Radio Receivers with Square-Law Detectors. J. Appl. Phys., 18,1947 , p. 383 .

13. Darling, D. A., and Siegert, A. J. F.: A Systematic Approach to a Class of Problems in the Theory of Noise and other Random Phenomena, I. IRE Trans. on Information Theory, IT-3, 1957, p. 32 .

14. Siegert, A. J. F.: A Systematic Approach to a Class of Problems in the Theory of Noise and other Random Phenomena, II, Examples. IRE Trans. on Information Theory, IT-3, 1957, p. 38 .

15. Ohta, M., and Koizumi, T.: Intensity Fluctuation of Stationary Random Noise Containing an Arbitrary Signal Wave. Proc. IEEE (Letters), 57, 6, June 1969, pp. 1231-1232.

16. Davenport, W., and Root, W. I.: Introduction to Random Signals and Noise. McGraw-Hill Book Co., Inc. New York, 1958.

17. Parzen, E.: Stochastic Processes. Holden-Day, Inc., San Francisco, Calif., 1962.

18. Papoulis, A.: Probability, Random Variables, and Stochastic Processes. McGraw-Hill Book Co., Inc., New York, 1965.

19. Van Trees, H. L.: Detection, Estimation, and Modulation Theory, Part I. John Wiley \& Sons, Inc., New York, 1968.

20. Lachs, G.: Theoretical Aspects of Mixtures of Thermal and Coherent Radiation. Phys. Rev., 138, 1965, pp. B1012-B1016.

21. Gradshteyn, I. S., and Ryzhik, I. M.: Table of Integrals Series and Products. Academic Press, New York, 1965.

22. Deutsch, R.: Nonlinear Transformations of Random Processes. Prentice-Hall, Englewood Cliffs, N. J., 1962.

23. Courant, R., and Hilbert, D.: Methods of Mathematical Physics. Vol. 1. Interscience, New York, 1953. 
24. Kendall, M. G., and Stuart, A.: The Advanced Theory of Statistics. Vol. 1. Griffin, London, 1963.

25. Klauder, J. R., and Sudarshan, E. C. G.: Fundamentals of Quantum Optics. W. A. Benjamin, Inc., New York, 1968.

26. Karp, S.: A Statistical Model for Radiation with Applications to Optical Communications. Ph. D. dissertation, University of Southern California, Dept. of Electrical Engineering, January 1967.

27. Liu, J. W-S.: Reliability of Quantum-Mechanical Communication Systems. Tech. Report 468, Research Laboratory of Electronics, Massachusetts Institute of Technology, December 31,1968 .

28. Fillmore, G. L., and Lachs, G.: Information Rates for Photocount Detection Systems. IEEE Trans. on Information Theory, IT-15, July 1969, pp. 465-468.

29. Reiffen, B., and Sherman, H.: An Optimum Demodulator for Poisson Processes: Photon Source Detectors. Proc. IEEE, October 1963, pp. 1316-1320.

30. Gagliardi, R. M., and Karp, S.: M-ary Poisson Detection and Optical Communications. IEEE Trans. on Comm. Tech., COM-17, No. 2, April 1969, pp. 208-216. 


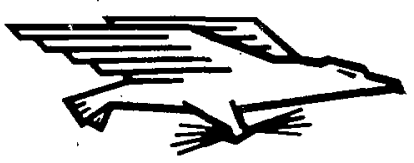

POSTAGE AND FEES PAID NATIONAL AERONAUTICS AND SPACE ADMINISTRATION

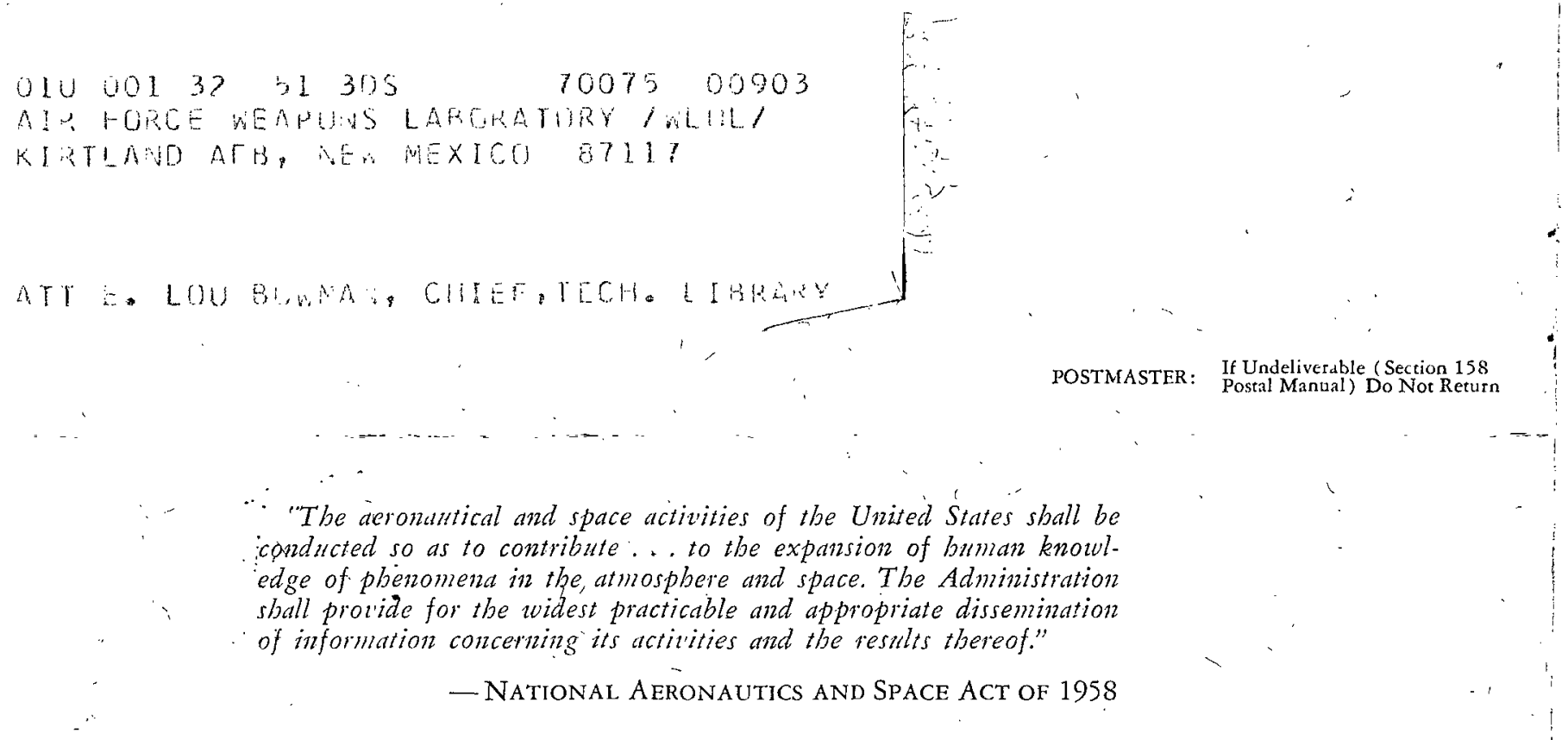

\section{NASA SCIENTIFIC AND TECHNICAL PUBLICATIONS}

TECHNICAL REPORTS: Scientific and technical information considered important, complete, and a lasting contribution to existing knowledge.

TECHNICAL NOTES: Information less broad in scope but nevertheless of importance as a contribution to existing knowledge.

TECHNICAL MEMORANDUMS:

Information receiving limited distribution because of preliminary data, security classification, or other reasons.

CONTRACTOR REPORTS: Scientific and technical information generated under a NASA contract or grant and considered an important contribution to existing knowledge.
TECHNICAL TRANSLATIONS: Information published in a foreign language considered to merit NASA distribution in English.

SPECIAL PUBLICATIONS: Information derived from or of value to NASA activities. Publications include conference proceedings, monographs, data compilations, handbooks, sourcebooks, and special bibliographies.

TECHNOLOGY UTILIZATION PUBLICATIONS: Information on technology used by NASA that may be of particular interest in commercial and other non-acrospace applications. Publications include Tech Briefs, Technology Utilization Reports and Notes, and Technology Surveys.

Details on the availability of these publications may be obtained from:

SCIENTIFIC AND TECHNICAL INFORMATION DIVISION

NATIONAL AERONAUTICS AND SPACE ADMINISTRATION

Washington, D.C. 20546 